\title{
Relationships for Moments of Generalized Order Statistics from Hjorth Distribution and Related Inference
}

\author{
Jagdish Saran, Kanika Verma ${ }^{*}$, Narinder Pushkarna
}

Department of Statistics, Faculty of Mathematical Sciences, University of Delhi, Delhi 110007, India

\section{ARTICLE INFO}

Article History

Received 02 Jun 2018

Accepted 02 Mar 2019

\section{Keywords}

Generalized order statistics

Hjorth distribution

single moments

product moments

recurrence relations

best linear unbiased estimators

type-II right censored samples

Mathematics Subject

Classification. 62G30

\section{ABSTRACT}

In this paper some recurrence relations satisfied by single and product moments of generalized order statistics from Hjorth distribution have been obtained. Then we use these results to compute the first two moments of order statistics for some specific values of the parameters. Further, we use the results on order statistics to obtain BLUEs of location and scale parameters based on type-II right censored samples.

(c) 2021 The Authors. Published by Atlantis Press B.V.

This is an open access article distributed under the CC BY-NC 4.0 license (http://creativecommons.org/licenses/by-nc/4.0/).

\section{INTRODUCTION}

Let $\left\{X_{n}, n \geq 1\right\}$ be a sequence of independent and identically distributed random variables with cumulative distribution function (cdf) $\mathrm{F}(\mathrm{x})$ and probability density function (pdf) $f(x)$.

Assume that $k>0, n \in N, n \geq 2, \tilde{m}=\left(m_{1}, m_{2}, \ldots, m_{n-1}\right) \in R^{n-1}, M_{r}=\sum_{j=r}^{n-1} m_{j}$ such that $\gamma_{r}=k+(n-r)+M_{r}>0$ for all $r \in\{1,2, \ldots, n-1\}$. Then $X(r, n, \tilde{m}, k), r=1,2, \ldots, n$, are called generalized order statistics if their joint pdf is given by

$$
\begin{aligned}
f_{X(1, n, \tilde{w}, k), \ldots, X(n, n, \tilde{w}, k)}\left(x_{1}, \ldots, x_{n}\right)= & k\left(\prod_{j=1}^{n-1} \gamma_{j}\right)\left(\prod_{i=1}^{n-1}\left(1-F\left(x_{i}\right)\right)^{m_{i}} f\left(x_{i}\right)\right) \\
& \times\left(1-F\left(x_{n}\right)\right)^{k-1} f\left(x_{n}\right), \\
& F^{-1}(0+)<x_{1} \leq \ldots \leq x_{n}<F^{-1}(1) .
\end{aligned}
$$

Choosing the parameters appropriately, models such as ordinary order statistics $\left(\gamma_{i}=n-i+1 ; i=1,2, \ldots, n\right.$, i.e., $m_{1}=m_{2}=\ldots m_{n-1}=$ $0, k=1), k$-th records values $\left(\gamma_{i}=k\right.$, i.e., $\left.m_{1}=m_{2}=\ldots=m_{n-1}=-1, k \in N\right)$, sequential order statistics $\left(\gamma_{i}=(n-i+1) \alpha_{i} ; \alpha_{1}, \alpha_{2}, \ldots, \alpha_{n}>\right.$ 0 ), order statistics with non-integral sample size $\left(\gamma_{i}=\alpha-i+1 ; \alpha>0\right)$, Pfeifer's record values $\left(\gamma_{i}=\beta_{i} ; \beta_{1}, \beta_{2}, \ldots, \beta_{n}>0\right)$ and progressively type-II right censored order statistics $\left(m_{i} \in N_{0}, k \in N\right)$ are obtained (cf. Kamps [1,2], Kamps and Cramer [3]).

The joint pdf of first $r$ generalized order statistics is given by

$$
\begin{aligned}
f_{X(1, n, \tilde{m}, k), \ldots, X(r, n, \tilde{m}, k)}\left(x_{1}, \ldots, x_{r}\right)= & c_{r-1}\left(\prod_{i=1}^{r-1}\left(1-F\left(x_{i}\right)\right)^{m_{i}} f\left(x_{i}\right)\right) \\
& \times\left(1-F\left(x_{r}\right)\right)^{k+(n-r)+M_{r}-1} f\left(x_{r}\right), \\
& F^{-1}(0+)<x_{1} \leq \ldots \leq x_{r}<F^{-1}(1) .
\end{aligned}
$$


We may consider two cases here:

Case I: $m_{1}=m_{2}=\ldots=m_{n-1}=m$.

Case II: $\gamma_{i} \neq \gamma_{j} ; i \neq j, i, j=1,2, \ldots, n-1$.

For Case I, the $r$-th generalized order statistic will be denoted by $X(r, n, m, k)$. The pdf of $X(r, n, m, k)$ is given by

$$
f_{X(r, n, m, k)}(x)=\frac{c_{r-1}}{(r-1) !}(1-F(x))^{\gamma_{r}-1} f(x) g_{m}^{r-1}(F(x)), \quad x \in R,
$$

and the joint pdf of $X(r, n, m, k)$ and $X(s, n, m, k), 1 \leq r<s \leq n$, is given by

$$
\begin{gathered}
f_{X(r, n, m, k), X(s, n, m, k)}(x, y)=\frac{c_{s-1}}{(r-1) !(s-r-1) !}[1-F(x)]^{m} f(x) g_{m}^{r-1}(F(x)) \\
\times\left[h_{m}(F(y))-h_{m}(F(x))\right]^{s-r-1}[1-F(y)]^{\gamma_{s}-1} f(y), x<y,
\end{gathered}
$$

where

$$
\begin{aligned}
& c_{r-1}=\prod_{j=1}^{r} \gamma_{j}, \quad \gamma_{r}=k+(n-r)(m+1), \quad r=1,2, \ldots, n, \\
& g_{m}(x)=h_{m}(x)-h_{m}(0), \quad x \in[0,1), \\
& h_{m}(x)= \begin{cases}\frac{-1}{m+1}(1-x)^{m+1} & , m \neq-1 \\
-\log (1-x) & , m=-1\end{cases}
\end{aligned}
$$

(cf. Kamps [1,2]).

For case II, $X(r, n, \tilde{m}, k)$ denotes the $r$-th generalized order statistic. The $\operatorname{pdf}$ of $X(r, n, \tilde{m}, k)$ is given by

$$
f_{X(r, n, \tilde{m}, k)}(x)=c_{r-1} f(x) \sum_{i=1}^{r} a_{i}(r)(1-F(x))^{\gamma_{i}-1}, \quad x \in R
$$

and the joint pdf of $X(r, n, \tilde{m}, k)$ and $X(s, n, \tilde{m}, k), 1 \leq r<s \leq \mathrm{n}$, is given by

$$
\begin{aligned}
& f_{X(r, n, \tilde{m}, k), X(s, n, \tilde{m}, k)}(x, y) \\
& =c_{s-1}\left\{\sum_{i=r+1}^{s} a_{i}^{(r)}(s)\left(\frac{1-F(y)}{1-F(x)}\right)^{\gamma_{i}}\right\}\left\{\sum_{i=1}^{r} a_{i}(r)(1-F(x))^{\gamma_{i}}\right\} \\
& \times \frac{f(x)}{1-F(x)} \frac{f(y)}{1-F(y)}, \quad x<y,
\end{aligned}
$$

where

$$
\begin{aligned}
& c_{r-1}=\prod_{i=1}^{r} \gamma_{i}, \quad \gamma_{i}=k+n-i+M_{i}, \quad r=1,2, \ldots, n, \\
& a_{i}(r)=\prod_{j(\neq i)=1}^{r} \frac{1}{\left(\gamma_{j}-\gamma_{i}\right)}, \quad 1 \leq i \leq r \leq n
\end{aligned}
$$

and

$$
a_{i}^{(r)}(s)=\prod_{j(\neq i)=r+1}^{s} \frac{1}{\left(\gamma_{j}-\gamma_{i}\right)}, \quad r+1 \leq i \leq s \leq n
$$

(cf. Kamps and Cramer [3]). 
Further, it can be easily proved that

$$
\begin{aligned}
& a_{i}(r)=\left(\gamma_{r+1}-\gamma_{i}\right) a_{i}(r+1), \\
& c_{r-1}=\quad \frac{c_{r}}{\gamma_{r+1}}, \\
& \sum_{i=1}^{r+1} a_{i}(r+1)= \\
& 0 \\
& \sum_{i=r+1}^{s} a_{i}^{(r)}(s)=
\end{aligned}
$$

Also, for $m_{i}=m_{j}=m$, it can be shown that

$$
\sum_{i=1}^{r} a_{i}(r)(1-F(x))^{\gamma_{i}}=\frac{(1-F(x))^{\gamma_{r}}}{(r-1) !} g_{m}^{r-1}(F(x)),
$$

and

$$
\begin{aligned}
\sum_{i=r+1}^{s} a_{i}^{(r)}(s)\left(\frac{1-F(y)}{1-F(x)}\right)^{\gamma_{i}}= & \frac{1}{(s-r-1) !}\left(\frac{1-F(y)}{1-F(x)}\right)^{\gamma_{s}} \\
& \times\left(\frac{1}{1-F(x)}\right)^{(m+1)(s-r-1)}\left[h_{m}(F(y))-h_{m}(F(x))\right]^{s-r-1} .
\end{aligned}
$$

Several authors like Kamps and Gather [4], Keseling [5], Cramer and Kamps [6], Ahsanullah [7], Pawlas and Szynal [8], Ahmad and Fawzy [9], Athar and Islam [10], Ahmad [11], Khan et al. [12], Khan et al. [13] and Saran and Pandey [14,15] have done some work on generalized order statistics. In this paper, in Section 3, we have established recurrence relations for single and product moments of generalized order statistics from Hjorth distribution for Case II only, i.e., for $\gamma_{i} \neq \gamma_{j} ; i \neq j, i, j=1,2, \ldots, n-1$. Then we use these results to compute means and variances of order statistics for some specific values of the parameters. Further, we use the results on order statistics to obtain BLUEs of location and scale parameters based on type-II right censored samples.

\section{HJORTH DISTRIBUTION}

A random variable $\mathrm{X}$ is said to have Hjorth distribution if its pdf is of the form

$$
f(x)=\frac{[(1+\beta x) \delta x+\theta] e^{-\frac{\delta x^{2}}{2}}}{(1+\beta x)^{1+\frac{\theta}{\beta}}}, \quad x \geq 0, \quad \beta, \delta, \theta>0
$$

and the cdf is of the form

$$
F(x)=1-\frac{e^{-\delta x^{2} / 2}}{(1+\beta x)^{\frac{\theta}{\beta}}} .
$$

Its characterizing differential equation is given by

$$
(1+\beta x) f(x)=[(1+\beta x) \delta x+\theta](1-F(x)) .
$$

More details on this distribution can be found in Hjorth [16].

The cdf of the location-scale parameter Hjorth distribution is given by

$$
F(x)=1-\frac{e^{-\delta\left(\frac{x-\mu}{\sigma}\right)^{2} / 2}}{\left(1+\beta\left(\frac{x-\mu}{\sigma}\right)\right)^{\frac{\theta}{\beta}}}, x \geq \mu, \mu \geq 0, \beta, \delta, \theta, \sigma>0 .
$$




\section{RECURRENCE RELATIONS FOR SINGLE AND PRODUCT MOMENTS OF GENERALIZED ORDER STATISTICS FROM HJORTH DISTRIBUTION FOR CASE II}

Theorem 3.1. For the distribution given in (10) and $n \in N, k \geq 1$,

$$
\begin{aligned}
& E\left[X^{i}(r, n, \tilde{m}, k)\right]+\beta E\left[X^{i+1}(r, n, \tilde{m}, k)\right] \\
& =\frac{\delta \gamma_{r}}{(i+2)}\left[E\left[X^{i+2}(r, n, \tilde{m}, k)\right]-E\left[X^{i+2}(r-1, n, \tilde{m}, k)\right]\right] \\
& +\frac{\delta \beta \gamma_{r}}{(i+3)}\left[E\left[X^{i+3}(r, n, \tilde{m}, k)\right]-E\left[X^{i+3}(r-1, n, \tilde{m}, k)\right]\right] \\
& +\frac{\theta \gamma_{r}}{(i+1)}\left[E\left[X^{i+1}(r, n, \tilde{m}, k)\right]-E\left[X^{i+1}(r-1, n, \tilde{m}, k)\right]\right] .
\end{aligned}
$$

Proof. From (5) and (12), we have

$$
\begin{aligned}
& E\left[X^{i}(r, n, \tilde{m}, k)\right]+\beta E\left[X^{i+1}(r, n, \tilde{m}, k)\right] \\
& =c_{r-1} \int_{0}^{\infty} x^{i}(1+\beta x) f(x) \sum_{i=1}^{r} a_{i}(r)[1-F(x)]^{\gamma_{i}-1} d x \\
& =c_{r-1} \int_{0}^{\infty} x^{i}\left[\delta x+\beta \delta x^{2}+\theta\right] \sum_{i=1}^{r} a_{i}(r)[1-F(x)]^{\gamma_{i}} d x \\
& =\delta c_{r-1} \int_{0}^{\infty} x^{i+1} \sum_{i=1}^{r} a_{i}(r)[1-F(x)]^{\gamma_{i}} d x \\
& +\beta \delta c_{r-1} \int_{0}^{\infty} x^{i+2} \sum_{i=1}^{r} a_{i}(r)[1-F(x)]^{\gamma_{i}} d x \\
& +\theta c_{r-1} \int_{0}^{\infty} x^{i} \sum_{i=1}^{r} a_{i}(r)[1-F(x)]^{\gamma_{i}} d x \\
& =\delta I_{i+1}+\beta \delta I_{i+2}+\theta I_{i}
\end{aligned}
$$

where

$$
\begin{aligned}
I_{i}= & c_{r-1} \int_{0}^{\infty} x^{i} \sum_{i=1}^{r} a_{i}(r)[1-F(x)]^{\gamma_{i}} d x \\
= & -\frac{c_{r-1}}{i+1} \int_{0}^{\infty} x^{i+1} \sum_{i=1}^{r-1} a_{i}(r)\left\{\left(\gamma_{r}-\gamma_{i}\right)-\gamma_{r}\right\}[1-F(x)]^{\gamma_{i}-1} f(x) d x \\
& +\frac{c_{r-1}}{i+1} \int_{0}^{\infty} x^{i+1} a_{r}(r) \gamma_{r}[1-F(x)]^{\gamma_{r-1}} f(x) d x \\
= & \frac{\gamma_{r}}{(i+1)}\left[E\left[X^{i}(r, n, \tilde{m}, k)\right]-E\left[X^{i+1}(r-1, n, \tilde{m}, k)\right]\right] .
\end{aligned}
$$

Putting the values of $I_{i}, I_{i+1}$ and $I_{i+2}$ from (16) into (15), we get the required result as given in (14).

Remark 3.1. Putting $m_{i}=m_{j}=m$ in (5) and using (8), the recurrence relation established in Theorem 3.1 reduces to the recurrence relation for single moments of generalized order statistics from Hjorth distribution for Case I, i.e., when $m_{1}=m_{2}=\ldots=m_{n-1}=m$.

Remark 3.2. If we take $k=1$ and $m_{i}=m_{j}=m=0$ in (14), we get the recurrence relation for single moments of order statistics from Hjorth distribution. Numerical computations for the means and variances of order statistics from Hjorth distribution for arbitrarily chosen values of $\beta, \delta$ and $\theta$ and for sample sizes $n=1(1) 10$ are given in Table 1.

Theorem 3.2. For $1 \leq r<s \leq n$ and $i, j \geq 0$,

$$
\begin{aligned}
& E\left[X^{i}(r, n, \tilde{m}, k) X^{j}(s, n, \tilde{m}, k)\right]+\beta E\left[X^{i}(r, n, \tilde{m}, k) X^{j+1}(s, n, \tilde{m}, k)\right] \\
& =\frac{\theta \gamma_{s}}{j+1}\left[E\left[X^{i}(r, n, \tilde{m}, k) X^{j}(s, n, \tilde{m}, k)\right]+E\left[X^{i}(r, n, \tilde{m}, k) X^{j+1}(s-1, n, \tilde{m}, k)\right]\right] \\
& +\frac{\delta \beta \gamma_{s}}{j+3}\left[E\left[X^{i}(r, n, \tilde{m}, k) X^{j+3}(s, n, \tilde{m}, k)\right]+E\left[X^{i}(r, n, \tilde{m}, k) X^{j+3}(s-1, n, \tilde{m}, k)\right]\right] \\
& +\frac{\delta \gamma_{s}}{j+2}\left[E\left[X^{i}(r, n, \tilde{m}, k) X^{j+2}(s, n, \tilde{m}, k)\right]+E\left[X^{i}(r, n, \tilde{m}, k) X^{j+2}(s-1, n, \tilde{m}, k)\right]\right]
\end{aligned}
$$


Table 1 Means and Variances of Order statistics from Hjorth distribution with parameters $\beta=2, \delta=3, \theta=4$.

\begin{tabular}{llllllll}
\hline $\boldsymbol{n}$ & $\boldsymbol{r}$ & $\mathrm{E}\left(\boldsymbol{X}_{r: n}\right)$ & $\operatorname{Var}\left(\boldsymbol{X}_{r: n}\right)$ & $\boldsymbol{n}$ & $\boldsymbol{r}$ & $\mathbf{E}\left(\boldsymbol{X}_{r: n}\right)$ & $\operatorname{Var}\left(\boldsymbol{X}_{r: n}\right)$ \\
\hline 1 & 1 & 0.275075 & 0.07296 & 8 & 1 & 0.032352 & 0.001111 \\
2 & 1 & 0.136533 & 0.019918 & 8 & 2 & 0.070568 & 0.002715 \\
2 & 2 & 0.413617 & 0.087615 & 8 & 3 & 0.116513 & 0.005054 \\
3 & 1 & 0.089588 & 0.008731 & 8 & 4 & 0.173041 & 0.008528 \\
3 & 2 & 0.230425 & 0.029067 & 8 & 5 & 0.244889 & 0.013882 \\
3 & 3 & 0.505213 & 0.091719 & 8 & 6 & 0.340947 & 0.02273 \\
4 & 1 & 0.066369 & 0.004787 & 8 & 7 & 0.481772 & 0.039734 \\
4 & 2 & 0.159242 & 0.014093 & 8 & 8 & 0.740519 & 0.089023 \\
4 & 3 & 0.301608 & 0.033907 & 9 & 1 & 0.02866 & 0.000868 \\
4 & 4 & 0.573081 & 0.092565 & 9 & 2 & 0.061889 & 0.002076 \\
5 & 1 & 0.052615 & 0.002992 & 9 & 3 & 0.100945 & 0.003768 \\
5 & 2 & 0.121387 & 0.008186 & 9 & 4 & 0.147648 & 0.006171 \\
5 & 3 & 0.216024 & 0.017581 & 9 & 5 & 0.204782 & 0.009662 \\
5 & 4 & 0.358664 & 0.036652 & 9 & 6 & 0.276974 & 0.014942 \\
5 & 5 & 0.626685 & 0.092176 & 9 & 7 & 0.372933 & 0.023554 \\
6 & 1 & 0.043547 & 0.002036 & 9 & 8 & 0.512868 & 0.040006 \\
6 & 2 & 0.097956 & 0.005302 & 9 & 9 & 0.768976 & 0.087862 \\
6 & 3 & 0.168248 & 0.010659 & 10 & 1 & 0.025722 & 0.000696 \\
6 & 4 & 0.263799 & 0.019939 & 10 & 2 & 0.055103 & 0.001636 \\
6 & 5 & 0.406097 & 0.03826 & 10 & 3 & 0.089032 & 0.002912 \\
6 & 6 & 0.670803 & 0.091282 & 10 & 4 & 0.128742 & 0.004661 \\
7 & 1 & 0.037129 & 0.001471 & 10 & 5 & 0.176008 & 0.007095 \\
7 & 2 & 0.082054 & 0.003696 & 10 & 6 & 0.233556 & 0.010572 \\
7 & 3 & 0.137711 & 0.007106 & 10 & 7 & 0.305919 & 0.01576 \\
7 & 4 & 0.208965 & 0.012496 & 10 & 8 & 0.401654 & 0.024145 \\
7 & 5 & 0.304925 & 0.021574 & 10 & 9 & 0.540672 & 0.040106 \\
7 & 6 & 0.446565 & 0.039202 & 10 & 10 & 0.794343 & 0.086733 \\
7 & 7 & 0.708176 & 0.090184 & & & & \\
\hline & & & & & & & \\
\hline
\end{tabular}

Proof. Using (6), we have

$$
\begin{aligned}
& E\left[X^{i}(r, n, \tilde{m}, k) X^{j}(s, n, \tilde{m}, k)\right]+\beta E\left[X^{i}(r, n, \tilde{m}, k) X^{j+1}(s, n, \tilde{m}, k)\right] \\
& =c_{s-1} \int_{0}^{\infty} \int_{x}^{\infty} x^{i} y^{j}(1+\beta y)\left\{\sum_{i=r+1}^{s} a_{i}^{(r)}(s)\left(\frac{1-F(y)}{1-F(x)}\right)^{\gamma_{i}}\right\}\left\{\sum_{i=1}^{r} a_{i}(r)(1-F(x))^{\gamma_{i}}\right\} \times \frac{f(x)}{1-F(x)} \frac{f(y)}{1-F(y)} d y d x \\
& =c_{s-1} \int_{0}^{\infty} x^{i} \sum_{i=1}^{r} a_{i}(r)(1-F(x))^{\gamma_{i}} \frac{f(x)}{1-F(x)} I(x) d x
\end{aligned}
$$

where

$$
I(x)=\int_{x}^{\infty} y^{j}(1+\beta y) \sum_{i=r+1}^{s} a_{i}^{(r)}(s)\left(\frac{1-F(y)}{1-F(x)}\right)^{\gamma_{i}} \frac{f(y)}{1-F(y)} d y .
$$

Using (12), we obtain

$$
\begin{aligned}
I(x) & =\int_{x}^{\infty} y^{j}[(1+\beta y) \delta y+\theta] \sum_{i=r+1}^{s} a_{i}^{(r)}(s)\left(\frac{1-F(y)}{1-F(x)}\right)^{\gamma_{i}} d y \\
& =\delta \int_{x}^{\infty} y^{j+1} \sum_{i=r+1}^{s} a_{i}^{(r)}(s)\left(\frac{1-F(y)}{1-F(x)}\right)^{\gamma_{i}} d y+\delta \beta \int_{x}^{\infty} y^{j+2} \sum_{i=r+1}^{s} a_{i}^{(r)}(s)\left(\frac{1-F(y)}{1-F(x)}\right)^{\gamma_{i}} d y \\
& +\theta \int_{x}^{\infty} y^{j} \sum_{i=r+1}^{s} a_{i}^{(r)}(s)\left(\frac{1-F(y)}{1-F(x)}\right)^{\gamma_{i}} d y \\
& =\delta I_{j+1}+\delta \beta I_{j+2}+\theta I_{j},
\end{aligned}
$$


where

$$
\begin{aligned}
& I_{j}=\int_{x}^{\infty} y^{j} \sum_{i=r+1}^{s} a_{i}^{(r)}(s)\left(\frac{1-F(y)}{1-F(x)}\right)^{\gamma_{i}} d y \\
& =\frac{1}{(j+1)} \int_{x}^{\infty} y^{j+1} \sum_{i=r+1}^{s} \gamma_{i} a_{i}^{(r)}(s)\left(\frac{1-F(y)}{1-F(x)}\right)^{\gamma_{i}} \frac{f(y)}{1-F(y)} d y \\
& =-\frac{1}{(j+1)} \int_{x}^{\infty} y^{j+1} \sum_{i=r+1}^{s-1} a_{i}^{(r)}(s)\left(-\gamma_{i}-\gamma_{s}+\gamma_{s}\right)\left(\frac{1-F(y)}{1-F(x)}\right)^{\gamma_{i}} \frac{f(y)}{1-F(y)} d y+\frac{1}{j+1} \int_{x}^{\infty} y^{j+1} a_{s}^{(r)}(s) \gamma_{s}\left(\frac{1-F(y)}{1-F(x)}\right)^{\gamma_{s}} \frac{f(y)}{1-F(y)} d y \\
& =-\frac{1}{(j+1)} \int_{x}^{\infty} y^{j+1} \sum_{i=r+1}^{s-1} a_{i}^{(r)}(s)\left(-\gamma_{i}+\gamma_{s}\right)\left(\frac{1-F(y)}{1-F(x)}\right)^{\gamma_{i}} \frac{f(y)}{1-F(y)} d y+\frac{\gamma_{s}}{(j+1)} \int_{x}^{\infty} y^{j+1} \sum_{i=r+1}^{s} a_{i}^{(r)}(s)\left(\frac{1-F(y)}{1-F(x)}\right)^{\gamma_{i}} \frac{f(y)}{1-F(y)} d y \\
& =-\frac{1}{(j+1)} \int_{x}^{\infty} y^{j+1} \sum_{i=r+1}^{s-1} a_{i}^{(r)}(s-1)\left(\frac{1-F(y)}{1-F(x)}\right)^{\gamma_{i}} \frac{f(y)}{1-F(y)} d y+\frac{\gamma_{s}}{(j+1)} \int_{x}^{\infty} y^{j+1} \sum_{i=r+1}^{s} a_{i}^{(r)}(s)\left(\frac{1-F(y)}{1-F(x)}\right)^{\gamma_{i}} \frac{f(y)}{1-F(y)} d y .
\end{aligned}
$$

Putting the values of $I_{j}, I_{j+1}$ and $I_{j+2}$ from (20) into (19) and then putting the value of $\mathrm{I}(\mathrm{x})$, so obtained, in (18), we get the required result as in Theorem 3.2.

Remark 3.5. Putting $m_{i}=m_{j}=m$ in (6) and using (8), and (9), the recurrence relation established in Theorem 3.2 reduces to the recurrence relation for single moments of generalized order statistics from Hjorth distribution for Case I, i.e., when $m_{1}=m_{2}=\ldots=$ $m_{n-1}=m$.

\section{BLUEs OF $\mu$ AND $\sigma$}

Let $X_{1: n} \leq X_{2: n} \leq \ldots \leq X_{n-c: n}, c=0,1, \ldots, n-1$, denote type-II right censored sample from the location-scale parameter Hjorth distribution in (13). Let us denote $Z_{r: n}=\left(X_{r: n}-\mu\right) / \sigma, E\left(Z_{r: n}\right)=\mu_{r: n}^{(1)}, 1 \leq r \leq(n-c)$, and $\operatorname{Cov}\left(Z_{r: n}, Z_{s: n}\right)=\sigma_{r, s: n}=\mu_{r, s: n}^{(1,1)}-\mu_{r: n}^{(1)} \mu_{s: n}^{(1)}, 1 \leq r<s \leq$ $(n-c)$. We shall use the following notations:

$$
\begin{gathered}
X=\left(X_{1: n}, X_{2: n}, \ldots, X_{n-c: n}\right)^{T} \\
\mu=\left(\mu_{1: n}, \mu_{2: n}, \ldots, \mu_{n-c: n}\right)^{T} \\
\mathbf{1}=(1,1, \ldots, 1)^{T} \\
\text { and } \sum=\left(\sigma_{r, s: n}\right), 1 \leq r, s \leq n-c .
\end{gathered}
$$

The BLUEs of $\mu$ and $\sigma$ are given by

$$
\mu^{*}=\left\{\frac{\mu^{T} \sum^{-1} \mu 1^{T} \sum^{-1}-\mu^{T} \sum^{-1} 1 \mu^{T} \sum^{-1}}{\left(\mu^{T} \sum^{-1} \mu\right)\left(1^{T} \sum^{-1} 1\right)-\left(\mu^{T} \sum^{-1} 1\right)^{2}}\right\} X=\sum_{r=1}^{n-c} a_{r} X_{r: n}
$$

and

$$
\sigma^{*}=\left\{\frac{1^{T} \sum^{-1} 1 \mu^{T} \sum^{-1}-1^{T} \sum^{-1} \mu 1^{T} \sum^{-1}}{\left(\mu^{T} \sum^{-1} \mu\right)\left(1^{T} \sum^{-1} 1\right)-\left(\mu^{T} \sum^{-1} 1\right)^{2}}\right\} X=\sum_{r=1}^{n-c} b_{r} X_{r: n},
$$

and the variances and covariance of these BLUEs are given by

$$
\operatorname{Var}\left(\mu^{*}\right)=\sigma^{2}\left\{\frac{\mu^{T} \sum^{-1} \mu}{\left(\mu^{T} \sum^{-1} \mu\right)\left(\mathbf{1}^{T} \sum^{-1} \mathbf{1}\right)-\left(\mu^{T} \sum^{-1} \mathbf{1}\right)^{2}}\right\}=\sigma^{2} V_{1},
$$




$$
\operatorname{Var}\left(\sigma^{*}\right)=\sigma^{2}\left\{\frac{\mathbf{1}^{T} \sum^{-1} \mathbf{1}}{\left(\mu^{T} \sum^{-1} \mu\right)\left(\mathbf{1}^{T} \sum^{-1} \mathbf{1}\right)-\left(\mu^{T} \sum^{-1} \mathbf{1}\right)^{2}}\right\}=\sigma^{2} V_{2}
$$

and

$$
\operatorname{Cov}\left(\mu^{*}, \sigma^{*}\right)=\sigma^{2}\left\{\frac{-\mu^{T} \sum^{-1} \mathbf{1}}{\left(\mu^{T} \sum^{-1} \mu\right)\left(\mathbf{1}^{T} \sum^{-1} \mathbf{1}\right)-\left(\mu^{T} \sum^{-1} \mathbf{1}\right)^{2}}\right\}=\sigma^{2} V_{3},
$$

respectively, (cf. Arnold et al. [17]).

Tables 2 and 3 display the coefficients of the BLUEs for type-II right censored samples of sizes $n=5(1) 10$ with $\beta=2, \delta=3$, $=4$ and different censoring cases $c=0(1)([n / 2]-1)$. The coefficients of the BLUEs in Tables 2 and 3 are checked by using the conditions

\begin{tabular}{|c|c|c|c|c|c|c|c|}
\hline \multirow{2}{*}{$\begin{array}{c}\beta, \delta, \theta \\
2,3,4\end{array}$} & \multirow[t]{2}{*}{$\mathbf{n}$} & \multirow[t]{2}{*}{ c } & \multicolumn{5}{|c|}{$a_{i}=1,2, \ldots,(n-c)$} \\
\hline & & & 1.185103 & -0.052238 & -0.041786 & -0.037652 & -0.053427 \\
\hline & & 1 & 1.252633 & -0.069672 & -0.057286 & -0.125675 & \\
\hline & \multirow[t]{4}{*}{6} & 0 & 1.156770 & -0.038448 & -0.030920 & -0.026106 & -0.024929 \\
\hline & & & -0.036367 & & & & \\
\hline & & 1 & 1.199844 & -0.047927 & -0.039163 & -0.033897 & -0.078857 \\
\hline & & 2 & 1.267204 & -0.062421 & -0.051839 & -0.152944 & \\
\hline & \multirow[t]{5}{*}{7} & 0 & 1.136374 & -0.029637 & -0.024218 & -0.020275 & -0.017906 \\
\hline & & & -0.017852 & -0.026486 & & & \\
\hline & & 1 & 1.166529 & -0.035450 & -0.029273 & -0.024876 & -0.022455 \\
\hline & & & -0.054475 & & & & \\
\hline & & 2 & 1.208535 & -0.043385 & -0.036193 & -0.031207 & -0.097750 \\
\hline & \multirow[t]{7}{*}{8} & 0 & 1.120713 & -0.023427 & -0.019590 & -0.016555 & -0.014305 \\
\hline & & & -0.013102 & -0.013503 & -0.020231 & & \\
\hline & & 1 & 1.143147 & -0.027284 & -0.022964 & -0.019592 & -0.01717 \\
\hline & & & -0.016030 & -0.040105 & & & \\
\hline & & 2 & 1.172097 & -0.032172 & -0.027250 & -0.023461 & -0.020845 \\
\hline & & & -0.068369 & & & & \\
\hline & & 3 & 1.214264 & -0.039128 & -0.033360 & -0.028998 & -0.112778 \\
\hline & \multirow[t]{8}{*}{9} & 0 & 1.108222 & -0.018806 & -0.016221 & -0.013874 & -0.011984 \\
\hline & & & -0.010652 & -0.010054 & -0.010624 & -0.016007 & \\
\hline & & 1 & 1.125638 & -0.021504 & -0.018611 & -0.016019 & -0.013967 \\
\hline & & & -0.012580 & -0.012074 & -0.030883 & & \\
\hline & & 2 & 1.146918 & -0.024749 & -0.021490 & -0.018607 & -0.016369 \\
\hline & & & -0.014927 & -0.050776 & & & \\
\hline & & 3 & 1.175679 & -0.029041 & -0.025302 & -0.022042 & -0.019571 \\
\hline & & & -0.079724 & & & & \\
\hline & \multirow[t]{10}{*}{10} & 0 & 1.098552 & -0.015890 & -0.013634 & -0.011818 & -0.010285 \\
\hline & & & 0.009068 & -0.008257 & -0.007988 & -0.008608 & -0.013003 \\
\hline & & 1 & 1.112506 & -0.017866 & -0.015396 & -0.013403 & -0.011740 \\
\hline & & & -0.010446 & -0.009629 & -0.009450 & -0.024576 & \\
\hline & & 2 & 1.128879 & -0.020154 & -0.017436 & -0.015243 & -0.013431 \\
\hline & & & -0.012053 & -0.011237 & -0.039324 & & \\
\hline & & 3 & 1.149846 & -0.023024 & -0.020000 & -0.017556 & -0.015564 \\
\hline & & & -0.014085 & -0.059616 & & & \\
\hline & & 4 & 1.178702 & -0.026892 & -0.023457 & -0.020681 & -0.018449 \\
\hline & & & -0.089223 & & & & \\
\hline
\end{tabular}

Table 2 Coefficients of the BLUE of location parameter. 
Table 3 Coefficients of the BLUE of scale parameter.

\begin{tabular}{|c|c|c|c|c|c|c|c|}
\hline \multirow{2}{*}{$\frac{\beta, \delta, \theta}{2,3,4}$} & \multirow{2}{*}{$\begin{array}{l}\mathbf{n} \\
5\end{array}$} & \multirow[t]{2}{*}{ c } & \multicolumn{5}{|c|}{$b_{i}=1,2, \ldots,(n-c)$} \\
\hline & & & -3.208033 & 0.757187 & 0.668368 & 0.688593 & 1.093885 \\
\hline & & 1 & -4.590649 & 1.114139 & 0.985722 & 2.490788 & \\
\hline & \multirow[t]{4}{*}{6} & 0 & -3.211420 & 0.644789 & 0.558759 & 0.524623 & 0.569260 \\
\hline & & & 0.913989 & & & & \\
\hline & & 1 & -4.293977 & 0.883014 & 0.765913 & 0.720441 & 1.924610 \\
\hline & & 2 & -5.937974 & 1.236757 & 1.075287 & 3.625931 & \\
\hline & \multirow[t]{5}{*}{7} & 0 & -3.220810 & 0.565699 & 0.490602 & 0.445028 & 0.437792 \\
\hline & & & 0.491353 & 0.790335 & & & \\
\hline & & 1 & -4.120641 & 0.739160 & 0.641423 & 0.582342 & 0.573543 \\
\hline & & & 1.584173 & & & & \\
\hline & & 2 & -5.342199 & 0.969912 & 0.842661 & 0.766453 & 2.763173 \\
\hline & \multirow[t]{7}{*}{8} & 0 & -3.231744 & 0.505453 & 0.441562 & 0.396476 & 0.373008 \\
\hline & & & 0.379760 & 0.436104 & 0.699380 & & \\
\hline & & 1 & -4.007270 & 0.638778 & 0.558222 & 0.501464 & 0.472092 \\
\hline & & & 0.480982 & 1.355732 & & & \\
\hline & & 2 & 4.985911 & 0.804020 & 0.703095 & 0.632261 & 0.596270 \\
\hline & & & 2.250264 & & & & \\
\hline & & 3 & -6.373787 & 1.032960 & 0.904193 & 0.814498 & 3.622136 \\
\hline & \multirow[t]{8}{*}{9} & 0 & -3.242010 & 0.456373 & 0.403847 & 0.361884 & 0.333970 \\
\hline & & & 0.323848 & 0.338372 & 0.394433 & 0.629282 & \\
\hline & & 1 & -3.926664 & 0.562450 & 0.497805 & 0.446202 & 0.411943 \\
\hline & & & 0.399652 & 0.417769 & 1.190842 & & \\
\hline & & 2 & -4.747246 & 0.608811 & 0.608811 & 0.546026 & 0.504558 \\
\hline & & & 0.490148 & 1.910109 & & & \\
\hline & & 3 & -5.829190 & 0.849026 & 0.752203 & 0.675246 & 0.624998 \\
\hline & & & 2.927717 & & & & \\
\hline & \multirow[t]{10}{*}{10} & 0 & -3.253575 & 0.418371 & 0.372557 & 0.334955 & 0.307007 \\
\hline & & & 0.290254 & 0.288326 & 0.307107 & 0.361682 & 0.573316 \\
\hline & & 1 & -3.868817 & 0.505504 & 0.450223 & 0.404843 & 0.371152 \\
\hline & & & 0.351006 & 0.348794 & 0.371600 & 1.065696 & \\
\hline & & 2 & -4.578786 & 0.604700 & 0.538706 & 0.484596 & 0.444504 \\
\hline & & & 0.420700 & 0.418543 & 1.667036 & & \\
\hline & & 3 & -5.467608 & 0.726402 & 0.647379 & 0.582682 & 0.534916 \\
\hline & & & 0.506848 & 2.469382 & & & \\
\hline & & 4 & -6.662882 & 0.886617 & 0.790576 & 0.712094 & 0.654412 \\
\hline & & & 3.619183 & & & & \\
\hline
\end{tabular}

Table 4 Variances and covariance of the BLUEs when $\mu=0$ and $\sigma=1$.

\begin{tabular}{cccccc}
\hline$\beta, \delta, \theta$ & $\mathbf{n}$ & $\mathbf{c}$ & $\operatorname{Var}\left(\mu^{*}\right)$ & $\operatorname{Var}\left(\sigma^{*}\right)$ & $\operatorname{Cov}\left(\mu^{*}, \sigma^{*}\right)$ \\
\hline $2,3,4$ & 5 & 0 & 0.003609 & 0.228627 & -0.011900 \\
& & 1 & 0.003864 & 0.335727 & -0.017130 \\
& 6 & 0 & 0.002368 & 0.183293 & -0.007828 \\
& & 1 & 0.002475 & 0.250810 & -0.010515 \\
& & 2 & 0.002641 & 0.349868 & -0.014574 \\
& 7 & 0 & 0.001669 & 0.152970 & -0.005532 \\
& & 1 & 0.001722 & 0.199847 & -0.007103 \\
& & 2 & 0.001795 & 0.261616 & -0.009227 \\
& & 0 & 0.001239 & 0.131258 & -0.004114 \\
& & 1 & 0.001268 & 0.165900 & -0.005116 \\
& & 2 & 0.001305 & 0.208513 & -0.006377 \\
& & 3 & 0.001359 & 0.267122 & -0.008158 \\
& & 0 & 0.000955 & 0.114940 & -0.003178 \\
& 1 & 0.000972 & 0.141689 & -0.003859 \\
& 2 & 0.000993 & 0.173057 & -0.004672 \\
& & 3 & 0.001022 & 0.213272 & -0.005741 \\
\hline
\end{tabular}


Table 4 Variances and covariance of the BLUEs when $\mu=0$ and $\sigma=1$. (Continued)

\begin{tabular}{cccccc}
\hline$\beta, \delta, \theta$ & $\mathbf{n}$ & $\mathbf{c}$ & $\operatorname{Var}\left(\mu^{*}\right)$ & $\operatorname{Var}\left(\sigma^{*}\right)$ & $\operatorname{Cov}\left(\mu^{*}, \sigma^{*}\right)$ \\
\hline & 10 & 0 & 0.000758 & 0.102222 & -0.002526 \\
& & 1 & 0.000769 & 0.123554 & -0.003010 \\
& 2 & 0.000781 & 0.147716 & -0.003567 \\
& & 3 & 0.000798 & 0.177205 & -0.004263 \\
& & 4 & 0.000820 & 0.215842 & -0.005196 \\
\hline
\end{tabular}

$$
\sum_{r=1}^{n-c} a_{r}=1
$$

and

$$
\sum_{r=1}^{n-c} b_{r}=0
$$

The variances and covariances of the BLUEs are presented in Table 4. We see that the variances of the BLUEs increase as the censoring level increases while the variances of the BLUEs decrease as the sample size increases. In addition, we see that the covariance of the BLUEs decreases as the censoring level increases while the covariance of the BLUEs increases as the sample size increases.

\section{CONFLICTS OF INTEREST}

The authors declare they have no conflicts of interest.

\section{AUTHORS' CONTRIBUTIONS}

All the three authors have equally contributed in the preparation of this research paper.

\section{Funding Statement}

We have solely funded the research by ourselves.

\section{ACKNOWLEDGMENTS}

The authors are grateful to the learned referees for their fruitful comments.

\section{REFERENCES}

1. U. Kamps, A Concept of Generalized Order Statistics, B.G. Teubner Stuttgart, Wiesbaden, Germany, 1995.

2. U. Kamps, J. Stat. Plann. Inference. 48 (1995), 1-23.

3. U. Kamps, E. Cramer, Statistics. 35 (2001), 269-280.

4. U. Kamps, U. Gather, Appl. Math. (Warsaw). 24 (1997), 383-391.

5. C. Keseling, Metrika. 49 (1999), 27-40.

6. E. Cramer, U. Kamps, J. Stat. Plann. Inference. 89 (2000), 79-89.

7. M. Ahsanullah, J. Stat. Plann. Inference. 85 (2000), 85-91.

8. P. Pawlas, D. Szynal, Commun. Stat.-Theory Meth. 30 (2001), 739-746.

9. A.A. Ahmad, M. Fawzy, J. Stat. Plann. Inference. 117 (2003), 241-249.

10. H. Athar, H.M. Islam, Metron. LXII (2004), 327-337.

11. A.A. Ahmad, J. Egypt. Math. Soc. 15 (2007), 117-128.

12. R.U. Khan, Z. Anwar, H. Athar, Aligarh J. Stat. 27 (2007), 69-79.

13. R.U. Khan, D. Kumar, H. Athar, Int. J. Stat. Syst. 5 (2010), 455-464.

14. J. Saran, A. Pandey, J. Appl. Stat. Sci. 13 (2004), 323-333.

15. J. Saran, A. Pandey, J. Stat. Theory Appl. 8 (2009), 383-391.

16. U. Hjorth, Technometrics. 17 (1980), 99-107.

17. B.C. Arnold, N. Balakrishnan, H.N. Nagaraja, A First Course in Order Statistics, John Wiley, New York, NY, USA, 1992. 